\title{
Synthesis and X-Ray Crystal Structure of Germaimines
}

\section{Anton Meller,* Günter Ossig, Walter Maringgele, Dietmar Stalke, Regine Herbst-Irmer, Stefanie Freitag and George M. Sheldrick}

\author{
Institut für Anorganische Chemie, Universität Göttingen, Tammannstraße 4, D-3400 Göttingen, Germany
}

Three new germaimines have been synthesized and two of them characterized by $\mathrm{X}$-ray diffraction; the $\mathrm{Ge}=\mathrm{N}$ bond lengths agree well with an $a b$ initio prediction for $\mathrm{H}_{2} \mathrm{Ge}=\mathrm{NH}$.

The $\mathrm{Ge}=\mathrm{N}$ distance in unsubstituted germaimine $\left(\mathrm{H}_{2} \mathrm{Ge}=\mathrm{NH}\right)$ has been predicted to be $169.5 \mathrm{pm}$ by ab initio calculation with double- $\zeta$ (DZ) plus polarization basis sets. ${ }^{1}$ Germaimines have been observed as transient species (and trapped by various substrates) since 1978 (see ref. 2 for a recent review). Only a few examples have been isolated and spectroscopically characterized within the last few years. ${ }^{3-8}$ Here we report the synthesis of three new germaimines prepared from highly sterically hindered diazagermylenes ${ }^{9}$ and 2,6-disubstituted phenylazides as shown in Scheme $1 . \dagger$ In the $15 \mathrm{~N}$ NMR spectrum of 3 two signals with $2: 1$ intensity ratio at $\delta=-295$ and -193 (rel. to $\mathrm{MeNO}_{2}$ ) have been detected.

Compounds 1-3 crystallized easily from hot THF (tetrahydrofuran) to give well-shaped amber crystals, which change from yellow at $-196{ }^{\circ} \mathrm{C}$ to red at $150{ }^{\circ} \mathrm{C}$. Compound 1 hydrolyses in air to give $\left[\left(2,6\left(\mathrm{Pr}^{\mathrm{i}}\right)_{2} \mathrm{C}_{6} \mathrm{H}_{3}\left(\mathrm{Me}_{3} \mathrm{Si}\right) \mathrm{N}\right]_{2} \mathrm{Ge}(\mathrm{OH})-\right.$ $\mathrm{NHC}_{6} \mathrm{H}_{3}-2,6\left(\mathrm{Pr}^{\mathrm{i}}\right)_{2} 4$ (from spectroscopic evidence). Likewise, methanol is added across the $(\mathrm{Ge}=\mathrm{N})$ double bond of 3 thus

$\dagger$ Preparative details and spectroscopic data: To a solution of the appropriate diazagermylene $(0.020 \mathrm{~mol})\{$ bis $[(2,6$-diisopropylphenyl)(trimethylsilyl)amino]germane(II) for 1, bis[(2,4,6-trimethylphenyl)(trimethylsilyl)aminolgermane(II) for 2 and 3 \} in $80 \mathrm{ml} \mathrm{THF}$ the equimolar amount of arylazide (2,6-diisopropylphenylazide for 1 and 2, 2,4,6-trimethylphenylazide for 3) dissolved in $40 \mathrm{ml}$ THF was added dropwise, with stirring, at ambient temperature and maintained at $25{ }^{\circ} \mathrm{C}$ for $2 \mathrm{~h}$. The clear, deep-red reaction mixtures was concentrated under reduced pressure, the amber crystals formed were filtered off and dried in vacuo. Satisfactory $\mathrm{C}, \mathrm{H}, \mathrm{N}$ elemental analyses were obtained for 1-3 and 5 .

1: Bis[(2,6-diisopropylphenyl)(trimethylsilyl)amino]-(2,6-diisopropylphenylimino)germane: m.p. $195^{\circ} \mathrm{C}$ (decomp.); yield $11.0 \mathrm{~g}$ $(74 \%) ; \mathrm{C}_{42} \mathrm{H}_{69} \mathrm{GeN}_{3} \mathrm{Si}_{2}$ (744.79); MS [m/z (\%)]: EI $745(20)\left[\mathrm{M}^{+}\right], 73$ (100); NMR (in $\left.\mathrm{C}_{6} \mathrm{D}_{6}\right): \delta\left({ }^{1} \mathrm{H}\right) 0.33\left(\mathrm{~s}, 18 \mathrm{H}, \mathrm{SiMe}_{3}\right) ; 1.06,1.28,1.32(3$ $\left.\times \mathrm{d},{ }^{3} J_{\mathrm{HH}} 6.6 \mathrm{~Hz}, 3 \times 6 \mathrm{H}, 6 \times \mathrm{CH}_{3}-\mathrm{Pr}^{\mathrm{i}}\right) ; 1.40-1.60(\mathrm{br}, 18 \mathrm{H}, 6 \times$ $\left.\mathrm{CH}_{3}-\mathrm{Pr}^{\mathrm{i}}\right) ; 2.90,3.80,4.06\left(3 \times\right.$ sept. $\left.3 \times 2 \mathrm{H}, 6 \times \mathrm{CH}-\mathrm{Pr}^{\mathrm{i}}\right) ; 6.70-7.10$, 7.24-7.32 $(2 \times$ br, $9 \mathrm{H}$, arom. $) ; \delta\left({ }^{13} \mathrm{C}\right) 3.57\left(\mathrm{SiMe}_{3}\right) ; 24.63,24.73$, $25.36,25.68$ (threefold intensity) (all $\left.\mathrm{CH}_{3}-\mathrm{Pr}^{\mathrm{i}}\right) ; 28.71,29.06,29.15$ (all CH-Pri); 120.05, 123.25, 124.93, 125.47, 127.08, 139.30, 141.67, $147.15,147.52,148.65$ (10 signals apparently due to non-equivalence of the $2 / 6$ and $3 / 5$ positions of the imino-bonded aromatic ring) (all arom. $) ; \delta\left({ }^{29} \mathrm{Si}\right) 16.2$.

2: Bis[(2,4,6-trimethylphenyl)(trimethylsilyl)amino]-(2,6-diisopropylphenylimino)germane: m.p. $162{ }^{\circ} \mathrm{C}$; yield $9.2 \mathrm{~g}(70 \%)$; $\mathrm{C}_{36} \mathrm{H}_{57} \mathrm{GeN}_{3} \mathrm{Si}_{2}$ (660.63); MS [m/z (\%)]: EI 661 (22) [ $\left.\mathrm{M}^{+}\right]$, $351(100) ;$ NMR (in $\left.\mathrm{C}_{6} \mathrm{D}_{6}\right): \delta\left({ }^{1} \mathrm{H}\right) 0.08\left(\mathrm{~s}, 18 \mathrm{H}, \mathrm{SiMe}_{3}\right) ; 1.50\left(\mathrm{~d},{ }^{3} J_{\mathrm{HH}} 6.8 \mathrm{~Hz}\right.$, $\left.12 \mathrm{H}, 4 \times \mathrm{CH}_{3}-\mathrm{Pr}^{\mathrm{i}}\right) ; 1.97\left(\mathrm{~s}, 12 \mathrm{H}, 4 \times a-\mathrm{CH}_{3}\right) ; 2.08\left(\mathrm{~s}, 6 \mathrm{H}, 2 \times p-\mathrm{CH}_{3}\right)$, 3.86 (sept., $\left.2 \mathrm{H}, 2 \times \mathrm{CH}-\mathrm{Pr}^{\mathrm{i}}\right) ; 6.46-6.52\left(\mathrm{~m}, 4 \mathrm{H}, \mathrm{C}_{6} \mathrm{H}_{2}\right) ; 7.05-7.13$, 7.28-7.34 $\left(2 \times \mathrm{m}, 3 \mathrm{H}, \mathrm{C}_{6} \mathrm{H}_{3}\right) ; \delta\left({ }^{13} \mathrm{C}\right) 2.12\left(\mathrm{SiMe}_{3}\right) ; 20.11\left(o-\mathrm{CH}_{3}\right)$; $20.72\left(p-\mathrm{CH}_{3}\right) ; 23.98\left(\mathrm{CH}_{3}-\mathrm{Pr}^{\mathrm{i}}\right) ; 29.38\left(\mathrm{CH}-\mathrm{Pr}^{\mathrm{i}}\right) ; 119.92,122.63$, $129.37,134.00,136.42,139.20,140.58,149.54$ (all arom.); $\delta\left({ }^{29} \mathrm{Si}\right)$ 14.1.

3: $\operatorname{Bis}[(2,4,6$-trimethylphenyl)(trimethylsilyl)amino]-(2,4,6-trimethylphenylimino)germane: m.p. $177{ }^{\circ} \mathrm{C}$; yield $8.54 \mathrm{~g}(69 \%)$; $\mathrm{C}_{33} \mathrm{H}_{51} \mathrm{GeN}_{3} \mathrm{Si}_{2}(618.55) ; \mathrm{MS}[\mathrm{m} / z(\%)]$ : EI $619(21)\left[\mathrm{M}^{+}\right], 351(100)$; NMR (in $\left.\mathrm{C}_{6} \mathrm{D}_{6}\right): \delta\left({ }^{1} \mathrm{H}\right) 0.11\left(\mathrm{~s}, 18 \mathrm{H}, \mathrm{SiMe}_{3}\right) ; 2.03\left(\mathrm{~s}, 18 \mathrm{H}, o-\mathrm{CH}_{3}\right.$ of mes + mes'); $2.33\left(\mathrm{~s}, 3 \mathrm{H}, p-\mathrm{CH}_{3}\right.$ of mes'/imino); $2.61\left(\mathrm{~s}, 6 \mathrm{H}, p-\mathrm{CH}_{3}\right.$ of mes/amino); $6.380,6.382\left(2 \mathrm{~s}, 4 \mathrm{H}, \mathrm{C}_{6} \mathrm{H}_{2}\right.$ of mes); 7.064, 7.066 (2s, $2 \mathrm{H}$, $\mathrm{C}_{6} \mathrm{H}_{2}$ of mes); $\delta\left({ }^{13} \mathrm{C}\right) 1.95\left(\mathrm{SiMe}_{3}\right) ; 20.66\left(o-\mathrm{CH}_{3}\right.$, mes/amino); 20.73 (o- $\mathrm{CH}_{3}$, mes'/imino); 21.04 ( $p-\mathrm{CH}_{3}$, mes); $21.06\left(p-\mathrm{CH}_{3}\right.$, mes'); $127.30,128.21,129.09,129.25,133.63,135.66,140.61,150.11,\left(\mathrm{C}_{6} \mathrm{H}_{2}\right.$ mes + mes' $\left.^{\prime}\right) ; \delta\left({ }^{29} \mathrm{Si}\right) 13.4 .-\delta\left({ }^{15} \mathrm{~N}\right)-\mathrm{NMR}(40 \%$ sol. in THF, standard $\mathrm{CH}_{3} \mathrm{NO}_{2}$ ext.): $-295(2 \mathrm{~N}),-193(1 \mathrm{~N})$.

$$
\begin{array}{r}
{\left[\mathrm{R}\left(\mathrm{Me}_{3} \mathrm{Si}\right) \mathrm{N}\right]_{2} \mathrm{Ge}+\mathrm{N}_{3} \mathrm{R}^{\prime} \stackrel{\mathrm{THF}, 2{ }^{\circ} \mathrm{C}}{-\mathrm{N}_{2}}\left[\mathrm{R}\left(\mathrm{Me}_{3} \mathrm{Si}\right) \mathrm{N}\right]_{2} \mathrm{Ge}=\mathrm{NR}^{\prime}} \\
\mathbf{1 : R}=\mathrm{R}^{\prime}=2,6\left(\mathrm{Pr}^{\mathrm{i}}\right)_{2} \mathrm{C}_{6} \mathrm{H}_{3} \\
\text { 2: } \mathrm{R}=2,4,6-\mathrm{Me}_{3} \mathrm{C}_{6} \mathrm{H}_{2}, \mathrm{R}^{\prime}=2,6\left(\mathrm{Pr}_{2}\right) \mathrm{C}_{6} \mathrm{H}_{3} \\
\mathbf{3 : R}=\mathrm{R}^{\prime}=2,4,6-\mathrm{Me}_{3} \mathrm{C}_{6} \mathrm{H}_{2} \\
\mathbf{3}+\mathrm{MeOH} \stackrel{\mathrm{THF}, 20^{\circ} \mathrm{C}}{\longrightarrow}\left[\mathrm{R}\left(\mathrm{Me}_{3} \mathrm{Si}\right) \mathrm{N}\right]_{2} \mathrm{Ge}(\mathrm{OMe})-\mathrm{NHR} \cdot \mathrm{THF} \\
\mathbf{5}\left(\mathrm{R}=2,4,6-\mathrm{Me}_{3} \mathrm{C}_{6} \mathrm{H}_{2}\right)
\end{array}
$$

Scheme 1

forming 5 which crystallizes with 1 mol of THF. $\ddagger$ The structures of 1 and 3 have been determined by single crystal $\mathrm{X}$-ray diffraction. $\S$ In these molecules, which are stable for months under dry nitrogen at room temperature, the $(\mathrm{Ge}=\mathrm{N})$ bond lengths are found to be 170.3(2) pm and 169.1(3) pm, which are in excellent agreement with the calculated value for germaimine. ${ }^{1}$ The $\mathrm{C}-\mathrm{N}=\mathrm{Ge}-\mathrm{N}$ torsion angles [1.5(5), $178.4(3)^{\circ} 1$ and $-1.5(5), 178.1(3)^{\circ} 3$ ] are consistent with a

\$ 4 m.p. $236^{\circ} \mathrm{C} ; \mathrm{C}_{42} \mathrm{H}_{71} \mathrm{GeN}_{3} \mathrm{OSi}_{2}(762.81) ; \mathrm{MS}[\mathrm{m} / z(\%)]: 763(2), 73$ (100); IR (KBr): $3583(v \mathrm{OH}), 3390(v \mathrm{NH})$.

5: Bis[2,4,6-trimethylphenyl)(trimethylsilyl)amino]methoxy $(2,4,6-$ trimethylphenylamino)germane was prepared by dropwise addition of a solution of $0.10 \mathrm{~g}(0.003 \mathrm{~mol}) \mathrm{MeOH}$ in $5 \mathrm{ml}$ THF to $0.93 \mathrm{~g}(0.0015$ $\mathrm{mol}$ ) of 3 in $10 \mathrm{ml}$ THF with stirring. After $1 \mathrm{~h}$ volatiles were removed in vacuo and the colourless residue recrystallized from THF: m.p. $130{ }^{\circ} \mathrm{C}$; yield $0.72 \mathrm{~g}(67 \%) ; \mathrm{C}_{38} \mathrm{H}_{63} \mathrm{GeN}_{3} \mathrm{O}_{2} \mathrm{Si}_{2}(722.70) ; \mathrm{MS}[\mathrm{m} / \mathrm{z}$ (\%)]: EI 651(8) [M - THF $]^{+}, 42(100)$.

NMR (in $\left.\mathrm{C}_{6} \mathrm{D}_{6}\right): \delta\left({ }^{1} \mathrm{H}\right) 0.30\left(\mathrm{~s}, 18 \mathrm{H}, \mathrm{SiMe}_{3}\right) ; 1.41(\mathrm{~m}, 4 \mathrm{H}, \mathrm{H}-3$ and $\mathrm{H}-4$ of THF); $1.95,2.06,2.14,2.18\left(4 \mathrm{~s}, 27 \mathrm{H}, o-\mathrm{CH}_{3}+p-\mathrm{CH}_{3}\right) ; 2.91(\mathrm{~s}$, $1 \mathrm{H}, \mathrm{NH}) ; 3.56(\mathrm{~m}, 4 \mathrm{H}, \mathrm{H}-2$ and $\mathrm{H}-5$ of THF); 3.91 (s, 3H, OMe); 6.60-6.80 (br, $\left.6 \mathrm{H}, \mathrm{C}_{6} \mathrm{H}_{2}\right)$; IR (in Nujol) $3350(\mathrm{vNH})$.

$\S$ Crystal data for 1: $\mathrm{C}_{42} \mathrm{H}_{69} \mathrm{GeN}_{3} \mathrm{Si}_{2}$, triclinic, space group $P \overline{1}$, $a=1144.6(2), b=1178.7(2), c=1728.6(3) \mathrm{pm}, \alpha=88.84(1)$, $\beta=80.67(1), \gamma=68.20(1)^{\circ}, U=2.13 \mathrm{~nm}^{3}, Z=2, D_{\mathrm{c}}=1.16 \mathrm{~g} \mathrm{~cm}^{-3}$, $\mu=0.80 \mathrm{~mm}^{-1}, F(000)=804$, crystal dimensions $0.5 \times 0.5 \times 0.6 \mathrm{~mm}$, 8471 reflections collected with $8<2 \theta<50^{\circ}, 7488$ unique and 6099 with $F>3 \sigma(F)$ used in the structural analysis. 3: $\mathrm{C}_{33} \mathrm{H}_{51} \mathrm{GeN}_{3} \mathrm{Si}_{2}$, monoclinic, space group $P 2_{1} / n, a=852.3(2), b=2961.1(4), c=$ $1382.1(2) \mathrm{pm}, \beta=100.30(2)^{\circ}, U=3.43 \mathrm{~nm}^{3}, Z=4, D_{\mathrm{c}}=1.20 \mathrm{~g} \mathrm{~cm}^{-3}$, $\mu=0.99 \mathrm{~mm}^{-1}, F(000)=1320$, crystal dimensions $0.4 \times 0.4 \times 0.6$ $\mathrm{mm}, 7086$ reflections collected with $8<2 \theta<50^{\circ}, 5991$ unique and 4589 with $F>3 \sigma(F)$ used in the structural analysis. Both data sets were collected on a Siemens-Stoe AED diffractometer using Mo-K $\alpha$ radiation $(\lambda=71.073 \mathrm{pm})$ at a temperature of $153 \mathrm{~K}$. Semiempirical absorption corrections were applied. The structures were solved by Patterson and Direct methods. All non-hydrogen atoms were refined anisotropically, and a riding model starting from calculated positions was employed for the hydrogen atoms. 4371 and 3563 parameters were refined with a weighting scheme $\left[w^{-1}=\sigma^{2}(F)+0.0002 F_{\mathrm{o}}^{2}\right] 1$ and $\left[w^{-1}=\sigma^{2}(F)+0.00015 F_{\mathrm{o}}^{2}\right] 3$. The refinement converged with $R$ $=0.041, R_{\mathrm{w}}=0.0371$ and $R=0.048, R_{\mathrm{w}}=0.0403$ and final difference electron density maxima of 0.511 and 0.423 and minima of -0.421 and $-0.4110^{-6} \mathrm{epm}^{-3} 3$. Atomic coordinates, bond lengths and angles, and anisotropic displacement parameters have been deposited at the Cambridge Crystallographic Data Centre. See Notice to Authors, Issue No. 1. 


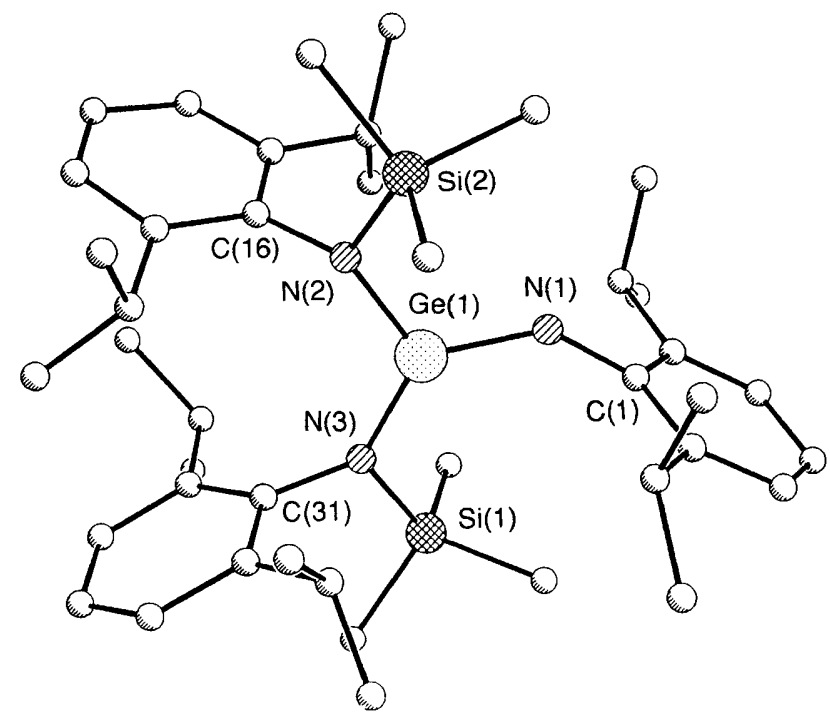

Fig. 1 Molecular structure of 1; selected bond distances (pm) and angles $\left({ }^{\circ}\right)$ : $\mathrm{Ge}(1)-\mathrm{N}(1) 170.3(2), \mathrm{Ge}(1)-\mathrm{N}(2) 181.9(3), \mathrm{Ge}(1)-\mathrm{N}(3)$ $182.6(3), \mathrm{N}(1)-\mathrm{C}(1) \quad 141.1(4), \mathrm{N}(2)-\mathrm{C}(16) \quad 145.3(3), \mathrm{N}(3)-\mathrm{C}(31)$ 145.5(3); N(1)-Ge(1)-N(2) 108.4(1), N(1)-Ge(1)-N(3) 130.9(1), $\mathrm{N}(2)-\mathrm{Ge}(1)-\mathrm{N}(3) 120.7(1)$

normal $\mathrm{p} \pi-\mathrm{p} \pi$ double bond. The difference between the mean $\mathrm{Ge}-\mathrm{N}$ and $\mathrm{Ge}=\mathrm{N}$ distances in these compounds is only $12 \mathrm{pm}$; for most pairs of atoms a double bond is about $20 \mathrm{pm}$ shorter than a single bond. In view of the $\mathrm{Ge}-\mathrm{N}$ distances reported here, $\left[\mathrm{GeN}\left(2,6-\mathrm{Pri}_{2} \mathrm{C}_{6} \mathrm{H}_{3}\right)\right]_{3}$ [mean Ge-N 185.9(2) pm] $]^{10}$ should be regarded as a $\mathrm{Ge}^{\mathrm{II}}$ derivative without significant multiple bonding.

Received, 16th May 1991; Com. 1/02326H

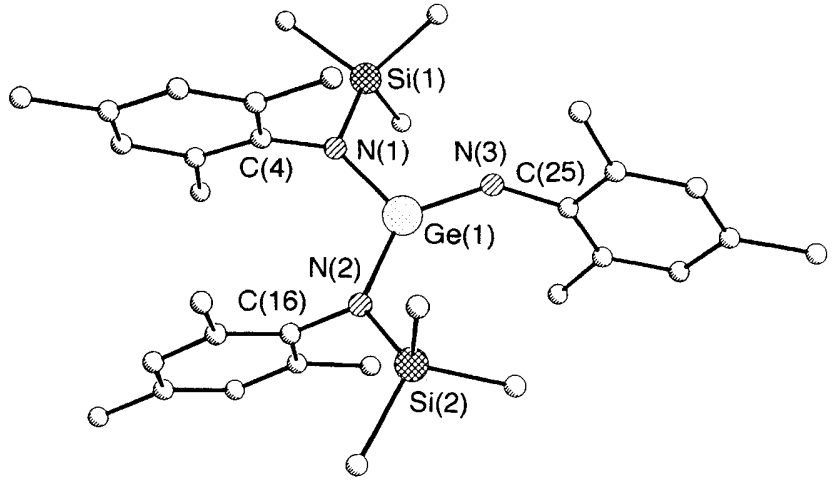

Fig. 2 Molecular structure of 3; selected bond distances (pm) and angles $\left({ }^{\circ}\right)$ : $\mathrm{Ge}(1)-\mathrm{N}(3)$ 169.1(3), $\mathrm{Ge}(1)-\mathrm{N}(1)$ 180.8(3), $\mathrm{Ge}(1)-\mathrm{N}(2)$ $182.8(3), \quad \mathrm{N}(3)-\mathrm{C}(25)$ 139.9(4), $\mathrm{N}(1)-\mathrm{C}(4) \quad 145.2(4), \mathrm{N}(2)-\mathrm{C}(16)$ 145.7(4); N(3)-Ge(1)-N(1) 110.6(1), N(3)-Ge(1)-N(2) 134.0(1), $\mathrm{N}(1)-\mathrm{Ge}(1)-\mathrm{N}(2) 115.4(1)$

\section{References}

1 G. Trinquier, J.-C. Barthelat and J. Satgé, J. Am. Chem. Soc., 1982, 104, 5931.

2 J. Satgé, J. Organomet. Chem., 1990, 400, 121

3 C. Glidewell, D. Lloyd, K. W. Lumbard and J. S. McKechnie, J. Chem. Soc., Dalton Trans., 1987, 2981.

4 C. Glidewell, D. Lloyd, K. W. Lumbard and J. S. McKechnie, Tetrahedron Lett., 1987, 28, 343.

5 J. Pfeiffer, W. Maringgele, M. Noltemeyer and A. Meller, Chem. Ber., 1989, 122, 245.

6 H. G. Ang and F. K. Lee, J. Chem. Soc., Chem. Commun., 1989, 310 .

7 H. G. Ang and F. K. Lee, J. Fluorine Chem., 1989, 43, 435.

8 T. Tsumuraya and W. Ando, Chem. Lett., 1989, 1043.

9 A. Meller and C. P. Graebe, Chem. Ber., 1985, 118, 2020.

10 R. A. Bartlett and P. P. Power, J. Am. Chem. Soc., 1990, 112, 3660 . 